\title{
Modulation of multiple pathways involved in the maintenance of neuronal function during aging by fisetin
}

\author{
Pamela Maher
}

Received: 17 July 2009/Accepted: 3 August 2009/Published online: 10 September 2009

(C) Springer-Verlag 2009

\begin{abstract}
Multiple factors have been implicated in the age-related declines in brain function. Thus, it is unlikely that modulating only a single factor will be effective at slowing this decline. A better approach is to identify small molecules that have multiple biological activities relevant to the maintenance of brain function. Over the last few years, we have identified an orally active, novel neuroprotective and cognition-enhancing molecule, the flavonoid fisetin. Fisetin not only has direct antioxidant activity but it can also increase the intracellular levels of glutathione, the major intracellular antioxidant. Fisetin can also maintain mitochondrial function in the presence of oxidative stress. In addition, it has anti-inflammatory activity against microglial cells and inhibits the activity of 5-lipoxygenase, thereby reducing the production of lipid peroxides and their pro-inflammatory by-products. This wide range of actions suggests that fisetin has the ability to reduce the age-related decline in brain function.
\end{abstract}

Keywords Oxidative stress - Glutathione - Nrf2 . Memory · Microglia

\section{Introduction}

Similar to other organs, brain function declines with age. Indeed, a decline in both cognitive and motor functions is

This article belongs to a special issue on Nutrients and Brain Health.

P. Maher $(\bowtie)$

The Salk Institute for Biological Studies,

10010 N. Torrey Pines Rd., La Jolla, CA 92037, USA

e-mail: pmaher@salk.edu one of the characteristics of normal aging, resulting in changes in learning and memory as well as deficits in balance and coordination. Furthermore, age is the single greatest risk factor for a variety of neurological disorders including Alzheimer's disease (AD). Since the average age in many Western countries is increasing, identifying approaches for reducing the effects of aging on brain function is taking on a new urgency. However, in order to choose among possible approaches, it is first necessary to identify the factors that contribute to the decrease in brain function with age. Among the factors that have been proposed are alterations in redox homeostasis, gene transcription, protein modification and processing, neurotrophic factor signaling, mitochondrial function and the immune response. Given this multiplicity of factors and the strong possibility that the relative importance of these factors will vary among individuals, approaches that are directed against a single target are unlikely to be generally useful. A better approach is to identify small molecules that have multiple biological activities that can impact the multiplicity of factors that are associated with the agerelated decrease in brain function.

One excellent source for these small molecules is the original pharmacopeia, plants. The polyphenolic flavonoids are widely distributed in fruits and vegetables and therefore regularly consumed in the human diet (for reviews see [32, $62,75])$. Flavonoids were historically characterized on the basis of their antioxidant and free radical scavenging effects. However, more recent studies have shown that flavonoids have a wide range of activities that could make them particularly effective for reducing the age-related deficits in brain function. In this article, I will discuss the multiple factors that are associated with the decline in brain function with age and show how one flavonoid, the flavone fisetin (Fig. 1), is able to impact each of these factors and therefore might hold promise for slowing or preventing this decline. 


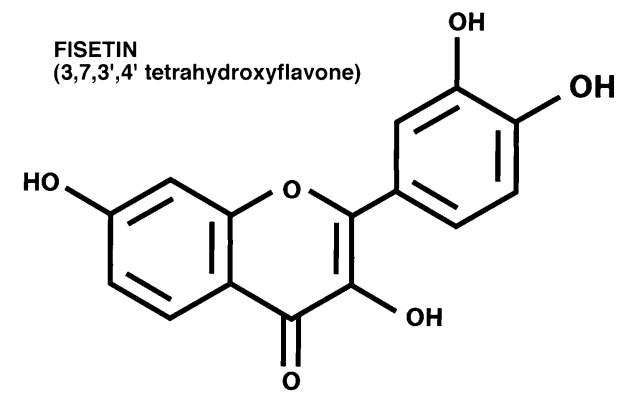

Fig. 1 Structure of fisetin

\section{Background}

Fisetin was originally identified in a screen for compounds that could prevent oxidative stress-induced nerve cell death [36]. Of the $\sim 30$ flavonoids tested in this study, only two, fisetin and quercetin, were able to maintain GSH levels in the presence of oxidative stress, indicating that this is not a common property of flavonoids. Further studies showed that fisetin also possessed neurotrophic activity, promoting the differentiation of PC12 cells via activation of the RasERK cascade [76]. Again, this was a property that distinguished fisetin from almost all of the other $\sim 30$ flavonoids tested. Only quercetin, isorhamnetin and luteolin showed some differentiation-inducing activity and they were all much less effective than fisetin. Together, these observations suggested that fisetin had multiple properties that might be able to contribute to the maintenance of nerve cell function.

Unlike many of the better studied flavonoids such as quercetin and luteolin, fisetin is not particularly abundant in fruits and vegetables. The highest levels $(160 \mu \mathrm{g} / \mathrm{g})$ are found in strawberries [2] with five to tenfold lower levels in apples and persimmons. Small amounts are also found in kiwi fruit, peaches, grapes, tomatoes, onions and cucumbers [33]. The bioavailability of fisetin from these sources has not been studied.

\section{Maintenance of redox homeostasis by fisetin}

\section{Antioxidant activity}

Over the years, a number of theories have been put forth to explain the mechanisms underlying the process of aging. One of the theories that has received the most attention and research support is the free radical theory of aging (for reviews see $[7,31,64] \# 1674)$. The current version of this theory proposes that there is an increase in the imbalance between pro-oxidants and antioxidants and, as a consequence, oxidative damage with aging which is the primary cause of the age-related declines in physiological function.
The oxidants arise from several sources and include both reactive oxygen (ROS) and reactive nitrogen (RNS) species. The main sources of ROS include mitochondrial respiration, lipid peroxidation and NADPH oxidase activity. Fortunately, cells contain an arsenal of antioxidant defenses including both antioxidant enzymes and small antioxidant molecules such as the endogenous antioxidant glutathione (GSH), as well as antioxidants derived from fruits and vegetables which can normally remove the ROS/ RNS generated by basic physiological functions. However, increasing age is associated with an imbalance between the production and removal of ROS/RNS resulting in oxidative stress and subsequent oxidative damage to proteins, lipids and DNA.

Although it is not entirely clear how relevant the antioxidant activity of flavonoids as measured in test tube assays is to their effects in vivo, fisetin is a relatively good antioxidant with a Trolox equivalent antioxidant capacity (TEAC) value of $\sim 3[36,54]$. Furthermore, fisetin was also shown to be very good at inhibiting lipid peroxidation and chelating iron [90]. An age-related increase in iron was found in human, rat and mouse brains [25] and was associated with markers of oxidant-mediated damage. All of these properties could contribute to the beneficial effects of fisetin on CNS cells.

\section{Maintenance of GSH}

GSH plays a central role in maintaining cellular redox homeostasis. A fairly large number of studies have shown age-dependent decreases in total GSH and/or reduced GSH levels in the brain (for reviews see [3, 25, 53]). In addition, age-dependent increases in glutathione disulfide (GSSG), the oxidized form of GSH have been observed in both mouse [72] and rat [67] brains. This, in turn, leads to an age-dependent decrease in the GSH/GSSG ratio, suggesting a significant alteration in the redox environment of the brain with age.

The age-related decreases in total GSH that are seen in many studies could be due to increased GSH consumption, decreased GSH production or some combination of the two. Increased consumption would be consistent with an increase in ROS production with age. However, recent studies suggest that decreased production also plays an important role in the decline of brain GSH with age. These studies have all shown a good correlation between decreases in the level of glutamate cysteine ligase (GCL) activity, the rate limiting enzyme in GSH biosynthesis, and decreases in GSH levels [50, 51, 63, 71, 77]. In addition, the decreases in GCL activity are correlated with decreases in the levels of GCL protein and/or mRNA of at least one of the two subunits $[50,51]$. A recent paper showed that the decrease in GCL levels, at least in liver, is due to a 
decrease in the level of $\mathrm{Nrf} 2$, the transcription factor involved in the induction of the genes encoding both chains of GCL [84]. Moreover, the low levels of Nrf2 in the livers of 24-28-month-old rats could be restored by treatment with lipoic acid, resulting in a restoration of both GCL activity and GSH levels. This suggests that the basic transcriptional response mechanism is still present in the old rats, but that for some reason the basal set point is turned down during aging. Although further research is clearly needed to determine if decreases in Nrf2 levels are also seen in the brain with aging, these findings suggest that compounds which can increase Nrf2 might be useful for maintaining redox homeostasis in the brain and thereby helping to maintain brain function.

Over the last few years, we have developed several in vitro models that can be used to identify small molecules that are able to maintain redox homeostasis in the presence of oxidative stress. Fisetin was first identified as a potential neuroprotective compound [36] using one of these models, oxidative glutamate toxicity (for review see [86]).

More recently, we have tested the effect of fisetin against peroxynitrite toxicity. Peroxynitrite levels increase during aging and may contribute to some of the nerve cell damage associated with normal aging [27]. In addition, peroxynitrite-mediated toxicity has been implicated in many age-related neurological disorders including ischemic stroke [26], AD, Parkinson's disease (PD), and amyotrophic lateral sclerosis (ALS) (reviewed in [87]). Although peroxynitrite itself is not a free radical, it is a uniquely damaging molecule because it can initiate strong oxidation reactions through decomposition into a hydroxyl radical and nitrogen dioxide [5]. It can also form a highly reactive nitroderivative in the presence of transition metals $[6,35]$ and interact directly with protein and non-protein thiol groups, leading to the depletion of cellular antioxidant defenses including GSH [70].

Using primary cultures of cortical neurons, in combination with the peroxynitrite generator SIN-1, we found an $\sim 50 \%$ decrease in both intracellular GSH levels and cellular viability that could be prevented by treatment with $10 \mu \mathrm{M}$ fisetin [12]. Similar results with fisetin were obtained when authentic peroxynitrite was used as the toxic insult. The protection by fisetin, as well as its ability to maintain GSH levels, was inhibited by treatment with buthionine sulfoximine (BSO), an inhibitor of GCL [30]. In contrast, BSO had no effect on the ability of glutathione monoethyl ester, a cell permeable form of GSH, to maintain GSH levels and protect the neurons from peroxynitrite toxicity. These data showed that BSO was not blocking the neuroprotective effect of fisetin through a toxic effect unrelated to GSH.
Mechanism of action

How does fisetin maintain GSH levels? In general, intracellular GSH levels are regulated by a complex series of mechanisms that include substrate availability and transport, rates of synthesis and regeneration, GSH utilization and GSH efflux to extracellular compartments (for review see [61]). Because glutamate and glycine occur at relatively high intracellular concentrations, cysteine is limiting for GSH synthesis in many types of cells, including nerve cells. In the extracellular environment, cysteine is readily oxidized to form cystine, so for most cell types, cystine transport mechanisms are essential to provide them with the cysteine needed for GSH synthesis. Cystine uptake in many types of cells is mediated by system $\mathrm{X}_{\mathrm{c}}^{-}$, a $\mathrm{Na}^{+}$-independent cystine/ glutamate antiporter [78]. System $\mathrm{X}_{\mathrm{c}}^{-}$is a member of the disulfide-linked heteromeric amino acid transporter family and consists of a light chain (xCT) that confers substrate specificity and a heavy chain $(4 \mathrm{~F} 2 \mathrm{hc})$ that is shared among a number of different amino acid transporters. The results with BSO suggested that fisetin increases GSH levels by either increasing cystine import and/or enhancing GCL activity.

Interestingly, both xCT [37] and GCL [15] are regulated at the transcriptional level by the transcription factor NF-E2-related factor 2 (Nrf2). Nrf2 binds to the antioxidant response element (ARE; also EpRE,) within the promoter of various genes, thereby regulating the inducible production of a variety of proteins involved in the protection of cells from oxidative stress as well as in the maintenance of redox homeostasis (for reviews see $[16,41,66])$. Treatment of cells with an Nrf2 inducer results in the accumulation and translocation of Nrf2 to the nucleus where it heterodimerizes with Maf family proteins to induce gene transcription. We have found that fisetin can increase the nuclear levels of Nrf2 in a variety of nerve cells including HT22 cells [54], retinal ganglion cells [56] and primary cortical neurons (Fig. 2). In primary cortical neurons treated with peroxynitrite [12], Nrf2 levels decreased significantly but treatment with fisetin was able to prevent this decrease. The peroxynitrite-mediated decreases in Nrf2 levels correlated with a decrease in the levels of both subunits of GCL, and GCL loss was also prevented by fisetin treatment. These findings are in agreement with earlier studies which showed that fisetin could increase the expression of various ARE-dependent genes in multiple non-neuronal cell lines [34, 65, 89] as well as rat C6 glioma cells [17]. The precise mechanisms whereby fisetin increases Nrf2 levels are still under investigation. 


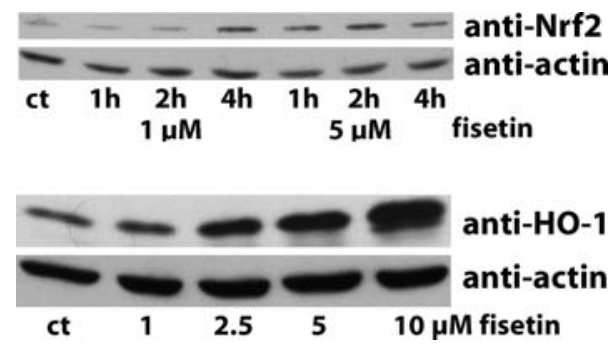

Fig. 2 Fisetin induces the expression of Nrf2, the ARE-specific transcription factor, and $\mathrm{HO}-1$, an Nrf-2-dependent protein. Rat primary cortical neurons ( 7 days in culture) were untreated $(c t)$ or treated with 1 or $5 \mu \mathrm{M}$ fisetin for 1,2 or $4 \mathrm{~h}(\mathrm{Nrf} 2)$ or with $1-10 \mu \mathrm{M}$ fisetin for $24 \mathrm{~h}(\mathrm{HO}-1)$. Nuclei were prepared (Nrf2) and equal amounts of protein were analyzed by SDS-PAGE and immunoblotting with Nrf2 antibodies or cell lysates were prepared and equal amounts of protein were analyzed by SDS-PAGE and immunoblotting with HO-1 antibodies. Immunoblotting with anti-actin is shown as a loading control. Similar results were obtained in three independent experiments

Fisetin can maintain mitochondrial function

Age-dependent changes in mitochondrial function are of particular interest as mitochondria are thought to play a key role in the aging process, for mitochondria are both a major source of intracellular oxidants as well as a target for the damaging effects of oxidants. In mammalian cells, mitochondria are the major source of energy in the form of ATP.

We have recently shown that fisetin is able to enhance basal ATP levels as well as maintain ATP levels in the presence of oxidative stress (Fig. 3). For these studies we used an in vitro ischemia model that we developed for the testing of compounds that might have therapeutic value for the treatment of stroke [58]. This model utilizes the toxin iodoacetic acid (IAA), a well known, irreversible inhibitor of the glycolytic enzyme glyceraldehyde 3-phosphate dehydrogenase, in combination with the HT22 nerve cell line. The changes seen following IAA treatment of nerve cells are very similar to changes that have been seen in animal models of ischemic stroke, and include alterations in membrane potential, breakdown of phospholipids, loss of ATP and GSH, and an increase in ROS. Using this model, we tested a variety of flavonoids and found that fisetin was highly effective at protecting the HT22 cells from IAA toxicity. Not only did fisetin prevent cell death but it stood out among the flavonoids tested as being able to maintain ATP levels in the presence of IAA. Further studies in the rabbit small clot embolism model of stroke demonstrated that fisetin could significantly improve the behavioral outcome when administered $5 \mathrm{~min}$ after the initiation of an embolic stroke [58]. Consistent with our animal data, fisetin was also shown to reduce ischemic damage and infarct volume

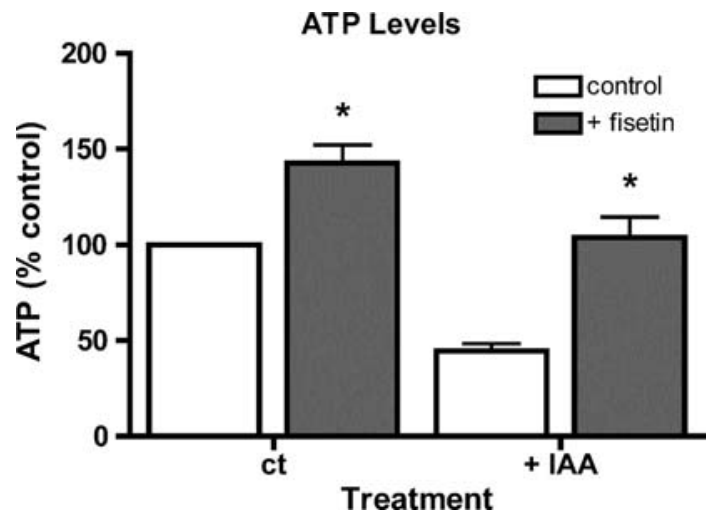

Fig. 3 Fisetin increases cellular ATP levels. HT22 cells were untreated $(c t)$ or treated with $20 \mu \mathrm{M}$ iodoacetic acid (+ IAA) to simulate ischemia for $2 \mathrm{~h}$ followed by $2 \mathrm{~h}$ in fresh medium alone in the absence (control) or presence (+ fisetin) of $10 \mu \mathrm{M}$ fisetin. Cell lysates were prepared and total ATP levels were measured using a chemiluminescent assay. Adapted from [58]

in the permanent focal middle cerebral artery occlusion model of stroke in rats [73].

Fisetin can enhance cognitive function

Normal human aging is associated with specific memory deficits including delayed recall of verbal information and declines in working memory, short-term recall, processing speed and spatial memory (for review see [94]). Although these deficits are distinct from those seen in neurological disorders such as $\mathrm{AD}$, they can still significantly impact the quality of life. Transcriptional profiling of the human frontal cortex [52] and rat hippocampus [10] showed agerelated decreases in genes involved in learning and memory. The transcription factor cAMP-response element binding protein (CREB) interacts with the cAMP-response element (CRE) in the promoter region of genes that encode proteins involved in the regulation of learning and memory (for reviews see $[13,60]$ ). A number of studies in a wide range of animal species have shown that CREB plays a key role in the formation of long-term memory (LTM) (for review see [88]). CREB is a constitutively nuclear protein whose activity is regulated by phosphorylation of both subunits of the homodimer [38]. Phosphorylation promotes the interaction of CREB with the transcriptional co-activator CREB binding protein (CBP) or its homolog p300, which stimulates the transcriptional activity of CREB. Several different kinases can phosphorylate CREB on Ser133 and positively regulate its transcriptional activity. These include protein kinase A (PKA), calmodulin dependent kinase IV, MNK1 and 2 and MSK1. The latter three kinases are all substrates of the MAP kinase ERK. Therefore, CREB activity can be regulated by the ERK signaling pathway. Using rat hippocampal slices, we 
demonstrated that $1 \mu \mathrm{M}$ fisetin could induce the rapid phosphorylation of CREB and that this phosphorylation was dependent on ERK activation since inhibitors of ERK activation also blocked CREB phosphorylation [57].

Given these results and the known associations between CREB and learning and memory, it was next asked whether fisetin could affect long-term potentiation (LTP) in the hippocampal slices. LTP is an in vitro assay that is considered to be a good model of how memory is formed at the cellular level [11]. Furthermore, age-related changes in cognitive function have been shown to correlate with impaired induction and maintenance of LTP [94]. Although fisetin had no effect on basal synaptic responses in the CA1 area of rat hippocampal slices [57], it induced LTP in slices exposed to a weak tetanic stimulus $(15$ pulses at $100 \mathrm{~Hz})$ that by itself failed to induce LTP. The facilitation of LTP by fisetin was dose dependent, with a maximal effect seen at $1 \mu \mathrm{M}$ and it persisted for at least $60 \mathrm{~min}$. Importantly, the facilitation of LTP by fisetin was blocked by two inhibitors of ERK activation, PD98059 and U0126. Together these data strongly support the hypothesis that ERKdependent CREB activation by fisetin is responsible for the facilitation of LTP by fisetin. Further support for this hypothesis comes from studies with the phosphodiesterase 4 inhibitor rolipram that enhances CREB phosphorylation by preventing the breakdown of cAMP. Rolipram also had no effect on basal synaptic responses in rat hippocampal slices but facilitated LTP induced by a weak tetanic stimulus in a manner very similar to fisetin [4]. However, in contrast to rolipram, fisetin did not increase cAMP levels in the hippocampal slices [57].

To determine if the biochemical and electrophysiological effects of fisetin seen in hippocampal slices could be translated into alterations in LTM in animals, we tested fisetin in mice using the object recognition task [9]. We chose this specific memory assay as our first test of fisetin on cognitive function because it had proven very effective for measuring CREB-dependent functions. In this test, during the training period, mice are presented with two identical objects, which they explore for a fixed time period. To test for LTM, the mice are presented 1 day later with two different objects, one of which was presented previously during the training and is thus familiar to the mice; the other object is new to them. The better the mice remember the familiar object, the more time they will spend exploring the novel object. To test the effects of fisetin in this memory task, it was administered orally to the mice before the start of the training period [57]. Rolipram, administered by injection, was used as a positive control. As shown in Fig. 4, fisetin significantly increased the time the mice spent exploring the novel object indicating a significant effect on LTM consistent with our celland tissue-based results.

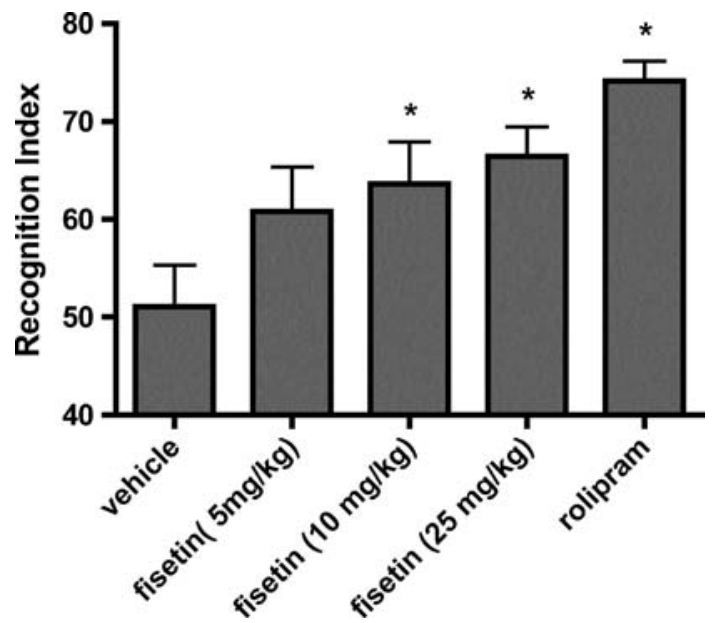

Fig. 4 Fisetin enhances long-term memory in young mice. The effect of different oral doses of fisetin on object recognition over a 10-min test period. Rolipram, injected intraperitoneally at $0.1 \mathrm{mg} / \mathrm{kg}$, served as a positive control. Data represent the mean \pm SEM of 10 mice/ treatment group. Data were analyzed by one-way ANOVA followed by post hoc comparisons with Fisher's test. Asterisk indicates significantly different from vehicle control $(P<0.02)$. Similar results were obtained in two independent, blinded experiments done by Psychogenics. From [57] (C) 2006 by The National Academy of Sciences of the USA

These studies were done with young animals so it was unclear whether fisetin would have similar effects in older animals. To begin to address this question, we fed mice fisetin for 10 months and tested their learning and memory using the morris water maze (MWM) at 12 months of age. The MWM is a test of spatial learning and is strongly correlated with hippocampal synaptic plasticity [91]. It can be used to assess both learning and memory deficits. In the standard test, the mice are required to find a submerged platform in a circular pool of opaque liquid (usually water with non-toxic white paint added) by relying on distal visual cues. Typically, they are given 4 trials/day over a period of 5-7 days and the time required for the mouse to find the platform (latency) is recorded. Twenty-four hours after the end of this learning phase, the mice are placed back in the pool but in this case the platform is no longer present. They are given a single, one min trial and the amount of time that they spend in the quadrant in which the platform was previously located relative to the time that they spend in the other three quadrants is recorded. As shown in Fig. 5, fisetin feeding slightly but non-significantly improved the learning and significantly improved the memory of older mice in the MWM relative to agematched controls fed a fisetin-free diet.

Among the fruits and vegetables where fisetin can be detected, the highest levels are found in strawberries [2]. Interestingly, supplementation of the diet of 19-month-old rats with a strawberry extract for 8 weeks resulted in 
MWM Escape Latency

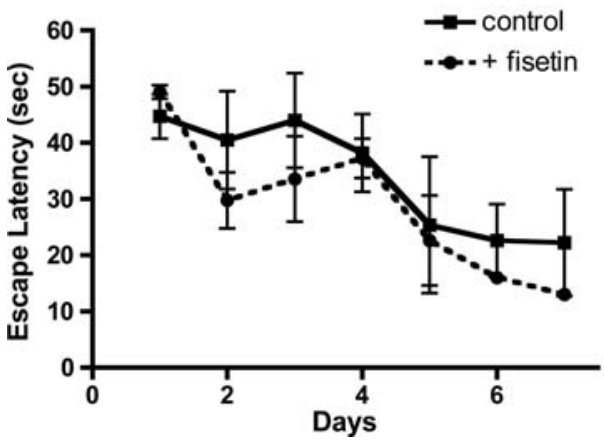

Fig. 5 Fisetin enhances long-term memory in older mice. Mice were fed fisetin $(500 \mathrm{mg} / \mathrm{kg}$ of food) for 10 months beginning at 2 months of age. Learning and memory were tested using the morris water maze $(M W M)$ [91]. Fisetin slightly but non-significantly enhanced learning in the mice as measured by the time taken to find a submerged platform over 7 days of training (left graph) and

enhanced performance in the MWM relative to rats fed a control diet [39]. Dietary supplementation with a strawberry extract also improved the performance of rats in the MWM in a rodent model of accelerated aging [81]. These results support our data with fisetin, and suggest that this flavonol could be useful for reducing at least some of the learning and memory deficits that accumulate with age.

\section{Anti-inflammatory effects of fisetin}

Microglia are the resident immune cell population of the CNS, comprising $10-15 \%$ of the total cell population (for reviews see [24, 29, 74]). They play important, protective roles in the CNS such as removing pathogens and promoting tissue regeneration after injury. However, activated microglia also produce a wide array of pro-inflammatory and cytotoxic factors including cytokines, ROS, excitatory neurotransmitters and eicosanoids that can promote nerve cell damage as well as impact cognitive function. For example, the cytokine IL1- $\beta$ can impair LTP [22]. Microglia are implicated in the pathogenesis of a variety of age-associated chronic neurological disorders including $\mathrm{AD}$ and PD. Importantly, microglial activation is also seen in the brains of healthy, aged animals [22] and is thought to play a role in the exaggerated immune response that is typical of the aged brain $[22,79]$. This can result in cognitive impairment and other behavioral deficits in response to stimuli that have little or no effect on these parameters in young animals. Thus, compounds that can modulate the activation of microglia in the aged brain and/or dampen their response to stimuli might have a significant benefit on brain function in the elderly.

A recent paper demonstrated that fisetin could reduce bacterial lipopolysaccharide (LPS)-induced microglial

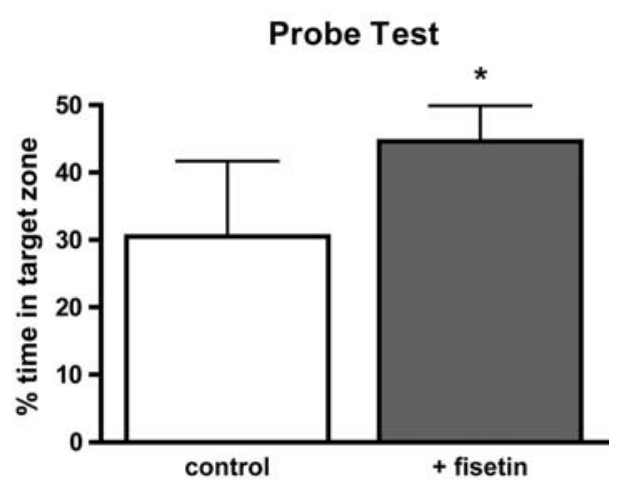

significantly enhanced memory as determined in the probe trial performed $24 \mathrm{~h}$ after the last training trial (right graph). Data represent the mean \pm SD of 4-6 mice/treatment group. Data for the latency trials were analyzed by ANOVA and data from the probe trial were analyzed by $t$ test. Asterisk indicates significantly different from control $(P<0.05)$

activation and neurotoxicity [96]. Using the BV-2 microglial cell line, it was shown that fisetin was very effective at blocking LPS-induced nitric oxide production, measured as accumulation of nitrite in the culture medium, with an $\mathrm{EC}_{50}$ of $\sim 7 \mu \mathrm{M}$. The same dose of fisetin also reduced LPS-induced increases in extracellular PGE2 levels as well as increases in the expression of the COX2, iNOS and interleukin- $1 \beta$ genes. Similar to the results obtained with other types of cells treated with fisetin and LPS [85, 92], the effects of fisetin on microglial activation appeared to be mediated by inhibition of LPS-stimulated NF- $\kappa$ B activation. Similar data were obtained with primary microglial cells isolated from the cerebral cortices of 1 day old mice. Importantly, these authors also showed neuroprotective effects of fisetin in a nerve/microglia co-culture system. In this assay, neuroblastoma cells were co-cultured with LPS-activated microglia with or without pre-treatment with fisetin. In the absence of fisetin, the LPS-activated microglia reduced the viability of the neuroblastoma cells by $\sim 50 \%$. However, following pre-treatment of the microglia with $\sim 7 \mu \mathrm{M}$ fisetin, the viability of the neuroblastoma cells was only reduced by $\sim 10 \%$. These results indicate that fisetin has anti-inflammatory activity and therefore might be effective in a variety of conditions involving the dysregulation of the immune system in the brain, including normal aging.

5-Lipoxygenase (5-LOX) metabolizes 20-carbon unsaturated fatty acids such as arachidonic acid, which are produced from membrane phospholipids by the action of phospholipases, to 5-hydroxyperoxyeicosatetraenoic acid (5-HPETE) followed by formation of the unstable intermediate leukotriene A4 (LTA4). LTA4 is then metabolized to leukotriene B4 or conjugated with GSH to form cysteinyl leukotrienes (for review see [68]). Via their binding 
to specific G-protein coupled receptors on target cells, leukotrienes can have pro-inflammatory effects. 5-LOX is expressed in the brain where its expression and activity is specifically increased in the hippocampus with age [18]. In addition, loss of 5-LOX was associated with a reduced beta amyloid peptide burden in a transgenic mouse model of AD [28] suggesting that inhibition of 5-LOX might have benefits in both normal aging and AD.

In earlier studies it was shown that fisetin is an effective inhibitor of 5-LOX activity in stimulated peritoneal leukocytes [49]. As shown in Fig. 6, we have confirmed and extended these studies. Fisetin inhibits 5-LOX from human peripheral blood mononuclear leukocytes (PBML) with an $\mathrm{IC}_{50}$ of $0.585 \mu \mathrm{M}$, a value which is as good or better than that of many known LOX inhibitors. Thus, these results suggest that an important activity of fisetin in the brain is the inhibition of 5-LOX activity. This action may contribute to both the neuroprotective and anti-inflammatory effects of fisetin and thereby promote the functional maintenance of the CNS during aging.

\section{Fisetin can enhance proteasome activity}

The ubiquitin-proteasome pathway mediates the majority of the proteolysis seen in the cytoplasm and nucleus of mammalian cells. As such it plays an important role in the regulation of a variety of physiological and pathophysiological processes (for reviews see [20, 43]). Several studies have shown that there is a specific decrease in proteasome activity in the hippocampus, cortex, striatum, globus pallidus and substantia nigra with aging in rodents $[40,95]$. In contrast, little or no change in proteasome activity is seen in the cerebellum and brain stem. These findings are consistent with studies that have

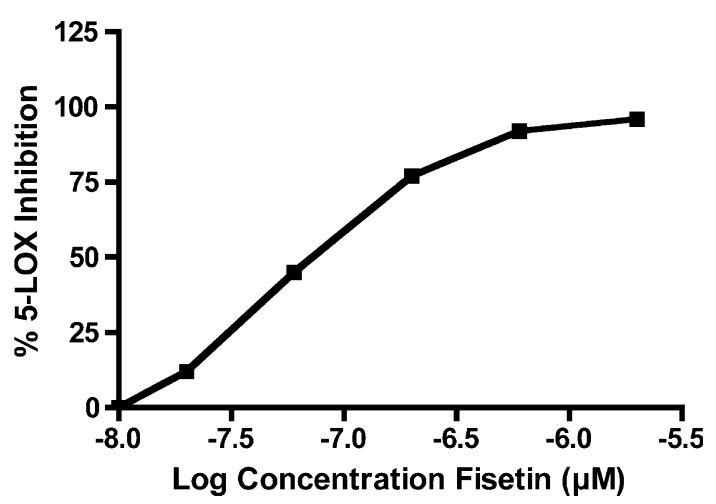

Fig. 6 Fisetin inhibits 5-LOX activity. Peripheral blood mononuclear leukocytes $(P B M L)$ were pre-incubated with the indicated doses of fisetin prior to the addition of arachidonic acid for 10-15 min. 5-LOX activity was determined from EIA quantitation of the 5-LOX product leukotriene B4 [14]. NDGA was used as a positive control for 5-LOX inhibition $\left(\mathrm{IC}_{50}=0.076\right)$ shown that proteasome activity is decreased in a variety of age-associated neurological disorders including $\mathrm{AD}$, $\mathrm{PD}$ and ALS $[8,19,21]$ and may contribute to disease progression. Interestingly, the age-related decreases in proteasome activity are generally not associated with decreased levels of overall proteasome immunoreactivity [23] suggesting that post-translational modifications to the proteasome are responsible for the decrease in activity. This decrease in proteasome activity is thought to play a critical role in the accumulation of abnormal and oxidized proteins. Indeed, microglia isolated from aged rodents show decreased proteasome activity and an impaired ability to degrade oxidized and glycated proteins [83].

Recently, we found that fisetin was able to modestly increase the chymotrypsin-like activity of the proteasome in primary cultures of cortical neurons and this contributed to the survival promoting effects of fisetin on these cells [55]. In further studies with the HT22 nerve cell line we have found that fisetin also increases the trypsin-like and caspase-like activities of the proteasome in a timeand dose-dependent manner (Fig. 7). Although it is not clear at this time how fisetin increases proteasome activity in nerve cells, the time- and substrate-dependence of its effects suggest that fisetin may have both direct and indirect actions on proteasome activity. Many of the protein components of the proteasome are transcriptionally regulated by $\mathrm{Nrf} 2$ [47] and Nrf2 inducers can increase proteasome activity in several different cell types [44-46]. However, preliminary data suggest that the regulation of proteasome activity by fisetin is likely to be more complex.

Enhancing proteasome activity by compounds such as fisetin could prove beneficial for reducing the CNS consequences of normal aging as well as treating neurological disorders. Although the increase in proteasome activity brought about by fisetin is modest, it is similar to the increases seen with several other compounds such as dithiolethione, 3-methylcholanthrene and $\beta$-napthoflavone $[44,46]$. Furthermore, dramatic increases in activity may not be compatible with the maintenance of normal cell function.

Consistent with the ability of fisetin to alter protein stability are two recent reports showing that fisetin can inhibit beta amyloid peptide fibril formation in a cell-free assay system [1, 42]. One of these studies [42] also showed that fisetin prevents extracellular beta amyloid peptide toxicity in the HT22 cells. Since beta amyloid peptide is thought to play a key role in the nerve cell loss that is the hallmark of $\mathrm{AD}$, these results suggest that fisetin may be able to reduce the burden of beta amyloid peptide through multiple mechanisms, including inhibition of aggregation and enhancement of degradation. 
Chymotrypsin-like Activity

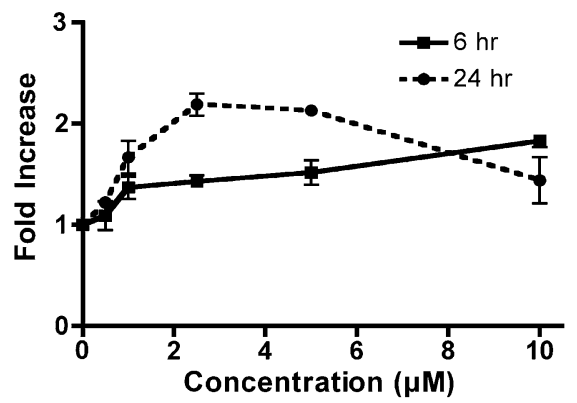

Caspase-like Activity

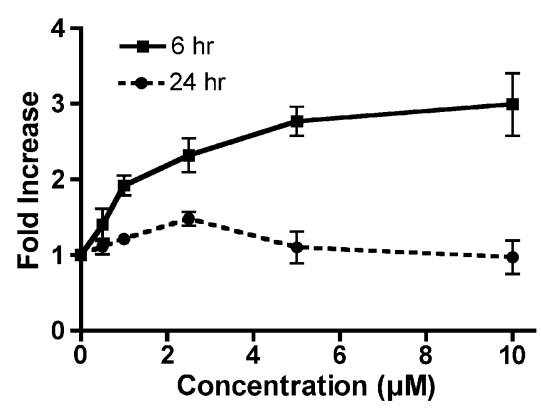

Trypsin-like Activity

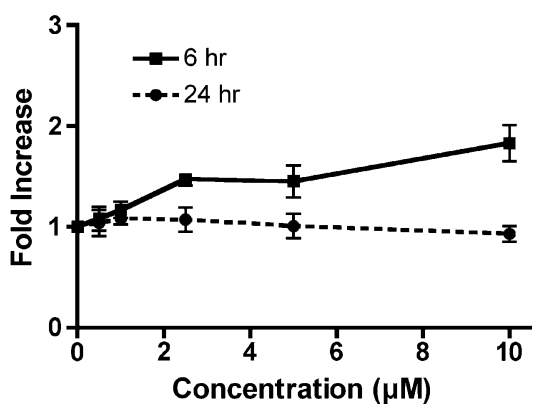

Fig. 7 Fisetin enhances proteasome activity in HT22 cells. HT22 cells were plated at $3 \times 10^{5}$ cells/dish in $60 \mathrm{~mm}$ dishes and grown for $24 \mathrm{~h}$. The cells were then left untreated or treated with the indicated concentrations of fisetin for 6 or $24 \mathrm{~h}$. The cells were harvested and assayed for proteasome activity as described [55] using fluorescent substrates for the chymotrypsin-like (Suc-LLVY-AMC), caspase-like (Z-LLG-AMC) and trypsin-like (Z-ARR-AMC) activity of the proteasome. Results are presented as mean $\pm \mathrm{SD}$ and are the average of four to five independent experiments

\section{Metabolism of fisetin}

Flavonoids are known to be extensively metabolized following oral consumption resulting in glucuronidated, sulfated and methylated metabolites (for reviews see [59, 69]). The metabolism of fisetin was recently characterized in rats following intravenous and oral administration [80]. Following both types of administration, the levels of free fisetin in serum declined rapidly while the levels of sulfated/glucuronidated fisetin increased. Following oral administration at $50 \mathrm{mg} / \mathrm{kg}$, the serum concentration of fisetin sulfates/glucuronides was maintained at $\sim 10 \mu \mathrm{M}$ for $>24 \mathrm{~h}$. These results are in sharp contrast to those obtained for $5 \mathrm{OH}$ and $7 \mathrm{OH}$ flavone [80] and baicalein [48] where the levels of free and conjugated flavonoid never exceeded $1 \mu \mathrm{M}$ following oral administration. When tested in an assay for antioxidant activity, the fisetin sulfates/glucuronides showed somewhat reduced but still significant activity as compared to free fisetin [80]. This result is consistent with a recent study on the effects of glucuronidation on the ability of several different flavonoids to protect nerve and lymphoid cells from oxidative stress-induced death [82]. Although the flavonoid glucuronides had generally higher $\mathrm{EC}_{50} \mathrm{~s}$ for protection as compared to their unmodified parent, they still showed excellent activity. Furthermore, circulating flavonoid sulfates/glucuronides can be cleaved to the free form in a tissue-specific manner if there is a local release of $\beta$-glucouronidases and/or sulfatases [69].

There is an ongoing debate about whether flavonoids such as fisetin can reach levels in the brain that are sufficient to affect neuronal function. Recently, using an in vitro model for blood brain barrier penetration [93], we found that fisetin exhibited high brain uptake potential (Table 1). Furthermore, following a single intraperitoneal injection, fisetin was detected in the brains of rats and this correlated
Table 1 Blood brain barrier penetration potential of fisetin

\begin{tabular}{lccll}
\hline Compound & A-B & B-A & Efflux & BBB penetration potential \\
\hline Fisetin & 18.0 & 1.33 & 0.1 & High \\
Caffeine $^{\mathrm{a}}$ & 20.2 & 22.2 & 1.1 & High \\
Atenolol $^{\mathrm{a}}$ & 0.13 & 0.25 & 1.9 & Low
\end{tabular}

The potential for blood brain barrier penetration was determined using MDR1-MDCK cell monolayers as described [93]. MDRMDCK cells plated at 60,000 cells $/ \mathrm{cm}^{2}$ on collagen-coated, microporous polycarbonate membranes in 12-well transwell plates were grown for 7-11 days. The quality of the monolayer was verified by TEER measurement $(>1,400)$. The test compounds at a final concentration of $5 \mu \mathrm{M}$ in HBSS were added to the apical or basolateral side and aliquots removed for analysis from the opposite side at $30 \mathrm{~min}$ intervals over $2 \mathrm{~h}$. The concentrations of the compounds in the samples were determined by LC/MS/MS. The potential for BBB penetration is viewed as high if Papp $\mathrm{A} \rightarrow \mathrm{B} \geq 3.0 \times 10^{-6} \mathrm{~cm} / \mathrm{s}$ and efflux $<3.0$, moderate if Papp $\mathrm{A} \rightarrow \mathrm{B} \geq 3.0 \times 10^{-6} \mathrm{~cm} / \mathrm{s}$ and $10>$ efflux $\geq 3.0$ and low if Papp $\mathrm{A} \rightarrow \mathrm{B} \geq 3.0 \times 10^{-6} \mathrm{~cm} / \mathrm{s}$ and efflux $\geq 10$ or Papp $\mathrm{A} \rightarrow \mathrm{B}<3.0 \times 10^{-6} \mathrm{~cm} / \mathrm{s}$. Results for caffeine and atenolol, CNS positive and negative compounds, respectively, are shown for comparison

${ }^{a}$ From Wang et al. [93]

with a significant reduction in cerebral damage in a stroke model [73]. Similarly, we have seen significant protection in a rabbit stroke model following intravenous injection of fisetin [58] and we found that oral administration of fisetin could enhance learning and memory in mice [57]. Thus, fisetin is able to affect neuronal function in vivo. Whether this is a direct effect of the unmodified fisetin molecule remains to be determined.

\section{Summary}

As discussed above, fisetin has positive effects on a number of common pathways associated with brain aging (Fig. 8). While the precise relationships among the 
Fig. 8 Fisetin affects multiple pathways implicated in brain aging. As discussed in this article or elsewhere, fisetin can increase neuronal function and survival through maintenance of redox homeostasis, activating neurotrophic factor signaling pathways [76], regulating protein homeostasis through effects on proteasome activity and protein aggregation and inhibiting inflammatory responses. Fisetin can also enhance cognitive function. Therefore, it has the potential to act as a multi-factorial drug for reducing the age-associated decline in brain function

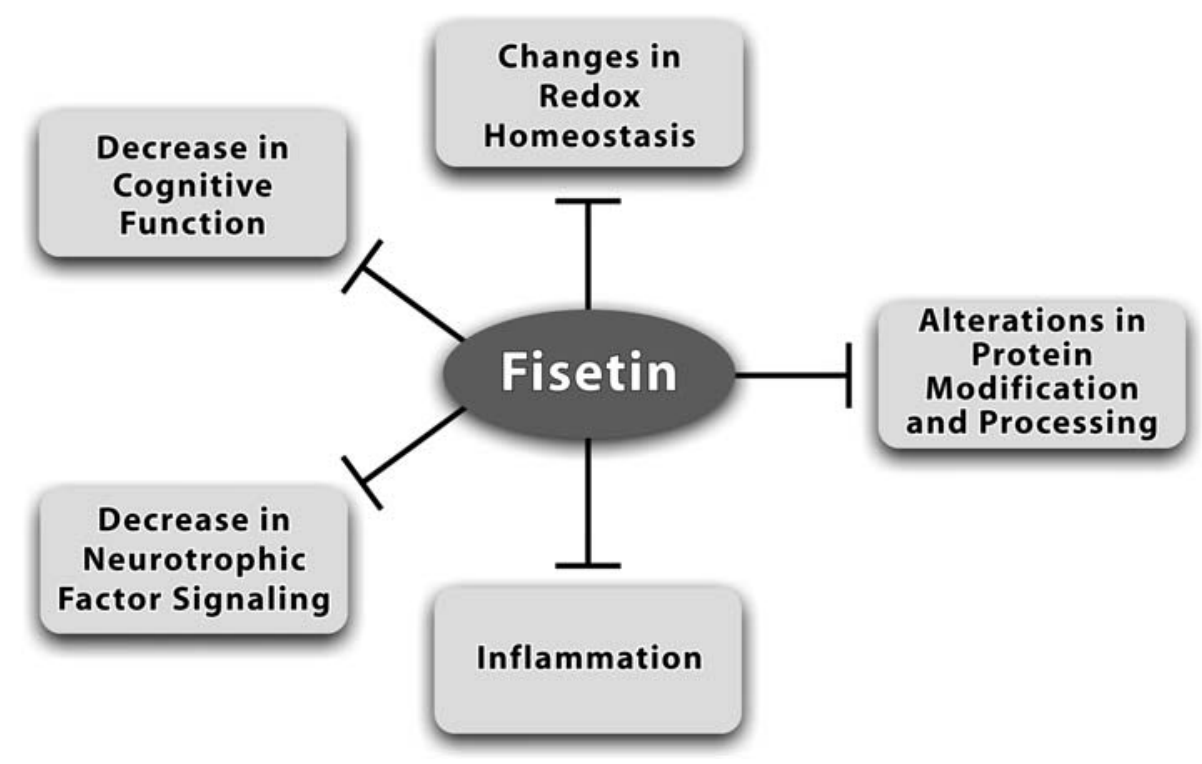

pleiotropic effects of fisetin on nerve cells are currently under investigation, this combination of actions suggests that fisetin has the potential to maintain neuronal function even in the presence of the diverse factors that contribute to the decline in brain function with age. Therefore, it might have therapeutic value as a multifactorial treatment to delay the decline in both cognitive and motor functions that is one of the characteristics of normal aging. These results also suggest that fisetin has the potential to reduce the impact of age-related neurodegenerative diseases, especially those associated with cognitive deficits such as AD. We are currently testing this hypothesis in feeding studies on control and $\mathrm{AD}$ transgenic mice.

Acknowledgments This work was supported by the Michael J. Fox Foundation and the Alzheimer's Association.

\section{References}

1. Akaishi T, Morimoto T, Shibao M, Watanabe S, Sakai-Kato K, Utsunomiya-Tate N, Abe K (2008) Structural requirement for the flavonoid fisetin in inhibiting fibril formation of amyloid $\beta$ protein. Neurosci Lett 444:280-285

2. Arai Y, Watanabe S, Kimira M, Shimoi K, Mochizuki R, Kinae N (2000) Dietary intakes of flavonols, flavones and isoflavones by Japanese women and the inverse correlation between quercetin intake and plasma LDL and cholesterol concentration. J Nutr 130:2243-2250

3. Ballatori N, Krance SM, Notenboom S, Shi S, Tieu K, Hammond CL (2009) Glutathione dysregulation and the etiology and progression of human diseases. Biol Chem 390:191-214

4. Barad M, Bourtchouladze R, Winder DG, Golan H, Kandel E (1998) Rolipram, a type IV-specific phosphodiesterase inhibitor, facilitates the establishment of long-lasting long-term potentiation and improves memory. Proc Natl Acad Sci USA 95:1502015025
5. Beckman JS, Beckman TW, Chen J, Marshall PA, Freeman BA (1990) Apparent hydroxyl radical production by peroxynitrite: implications for endothelial injury from nitric oxide and superoxide. Proc Natl Acad Sci USA 87:1620-1624

6. Beckman JS, Ischiropoulos H, Zhu L, van der Woerd M, Smith C, Chen J, Harrison J, Martin JC, Tsai M (1992) Kinetics of superoxide dismutase- and iron-catalyzed nitration of phenolics by peroxynitrite. Arch Biochem Biophys 298:438-445

7. Beckman KB, Ames BN (1998) The free radical theory of aging matures. Physiol Rev 78:547-581

8. Betarbet R, Sherer TB, Greenamyre JT (2005) Ubiquitin-proteasome system and Parkinson's disease. Exp Neurol 191:S17S27

9. Bevins RA, Besheer J (2006) Object recognition in rats and mice: a non-trial non-matching-to-sample learning task to study 'recognition memory'. Nat Protoc 1:1306-1311

10. Blalock EM, Chen K-C, Sharrow K, Herman JP, Porter NM, Foster TC, Landfield PW (2003) Gene microarrays in hippocampal aging: statistical profiling identifies novel processes correlated with cognitive impairment. J Neurosci 23:3807-3819

11. Bliss TVP, Collingridge GL (1993) A synaptic model of memory: long-term potentiation in the hippocampus. Nature 361:31-39

12. Burdo J, Schubert D, Maher P (2008) Glutathione production is regulated via distinct pathways in stressed and non-stressed cortical cultures. Brain Res 1189:12-22

13. Carlezon WA, Duman RS, Nestler EJ (2005) The many faces of CREB. Trends Neurosci 28:436-445

14. Carter GW, Young PR, Albert DH, Bouska J, Dyer R, Bell RL, Summers JB, Brooks DW (1991) 5-Lipoxygenase inhibitory activity of zileuton. J Pharmacol Exp Ther 256:929-937

15. Chan JY, Kwong M (2000) Impaired expression of glutathione synthetic enzyme genes in mice with targeted deletion of the Nrf2 basic-leucine zipper protein. Biochim Biophys Acta 1517:19-26

16. Chen C, Kong ANT (2004) Dietary chemopreventive compounds and ARE/EpRE signaling. Free Radic Biol Med 36:1505-1516

17. Chen TJ, Jeng JY, Lin CW, Wu CY, Chen YC (2006) Quercetin inhibition of ROS-dependent and -independent apoptosis in rat glioma C6 cells. Toxicology 223:113-126

18. Chinnici CM, Yao Y, Practico D (2007) The 5-lipoxygenase enzymatic pathway in the mouse brain: young versus old. Neurobiol Aging 28:1457-1462 
19. Chung KKK, Dawson VL, Dawson TM (2001) The role of the ubiquitin-proteasomal pathway in Parkinson's disease and other neurodegenerative disorders. Trends Neurosci 24:S7-S14

20. Ciechanover A (2005) Proteolysis: from the lysosome to ubiquitin and the proteasome. Nat Rev Mol Cell Biol 6:79-86

21. Ciechanover A, Brundin P (2003) The ubiquitin proteasome system in neurodegenerative diseases: sometimes the chicken, sometimes the egg. Neuron 40:427-446

22. Dilger RN, Johnson RW (2008) Aging, microglial priming, and the discordant central inflammatory response to signals from the peripheral immune system. J Leukoc Biol 84:932-939

23. Ding Q, Keller JN (2001) Proteasomes and proteasome inhibition in the central nervous system. Free Radic Biol Med 31:574-584

24. Dringen R (2005) Oxidative and antioxidative potential of brain microglial cells. Antioxid Redox Signal 7:1223-1233

25. Droge W, Schipper HM (2007) Oxidative stress and aberrant signaling in aging and cognitive decline. Aging Cell 6:361-370

26. Eliasson MJL, Huang Z, Ferrante RJ, Sasamata M, Molliver ME, Snyder SH, Moskowitz MA (1999) Neuronal nitric oxide synthase activation and peroxynitrite formation in ischemic stroke linked to neuronal damage. J Neurosci 19:5910-5918

27. Farooqui T, Farooqui AA (2008) Aging: an important factor for the pathogenesis of neurodegenerative diseases. Mech Ageing Dev 130:203-215

28. Firuzi O, Zhuo J, Chinnici CM, Wisniewski T, Pratico D (2008) 5-Lipoxygenase gene disruption reduces amyloid-beta pathology in a mouse model of Alzheimer's disease. FASEB J 22:11691178

29. Garden GA, Moller T (2006) Microglia biology in health and disease. J Neuroimmune Pharmacol 1:127-137

30. Griffith OW, Meister A (1979) Potent and specific inhibition of glutathione synthesis by buthionine sulfoximine (S-n-butyl homocysteine sulfoximine). J Biol Chem 254:7558-7560

31. Harman D (1956) Aging: a theory based on free radical and radiation chemistry. J Gernontol 11:298-300

32. Heim KE, Tagliaferro AR, Bobilya DJ (2002) Flavonoid antioxidants: chemistry, metabolism and structure-activity relationships. J Nutr Biochem 13:572-584

33. Hendler SS (2008) PDR for nutritional supplements, 2nd edn. Thomson Reuters, Cambridge

34. Hou D-X, Fukuda M, Johnson JA, Miyamori K, Ushikai M, Fujii M (2001) Fisetin induces transcription of NADPH:quinone oxidoreductase gene through an antioxidant responsive elementinvolved activation. Int J Oncol 18:1175-1179

35. Ischiropoulos H, Zhu L, Chen J, Tsai M, Martin JC, Smith CD, Beckman JS (1992) Peroxynitrite-mediated tyrosine nitration catalyzed by superoxide dismutase. Arch Biochem Biophys 298:431-437

36. Ishige K, Schubert D, Sagara Y (2001) Flavonoids protect neuronal cells from oxidative stress by three distinct mechanisms. Free Radic Biol Med 30:433-446

37. Ishii T, Itoh K, Takahashi S, Sato H, Yanagawa T, Yasutake K, Bannai S, Yamamoto M (2000) Transcription factor Nrf2 coordinately regulates a group of oxidative stress-inducible genes in macrophages. J Biol Chem 275:16023-16029

38. Johannessen M, Delghandi MP, Moens U (2004) What turns CREB on? Cell Signal 16:1211-1227

39. Joseph JA, Shukitt-Hale B, Denisova NA, Bielinski D, Martin A, McEwen JJ, Bickford PC (1999) Reversals of age-related declines in neuronal signal transduction, cognitive, and motor behavioral deficits with blueberry, spinach, or strawberry dietary supplementation. J Neurosci 19:8114-8121

40. Keller JN, Hanni KB, Markesbery WR (2000) Possible involvement of proteasome inhibition in aging: implications for oxidative stress. Mech Ageing Dev 113:61-70
41. Kensler TW, Wakabayashi N, Biswal S (2007) Cell survival responses to environmental stresses via the Keap1-Nrf2-ARE pathway. Annu Rev Pharmacol Toxicol 47:89-116

42. Kim H, Park B-S, Lee K-G, Choi CY, Jang SS, Kim Y-H, Lee S-E (2005) Effects of naturally occurring compounds on fibril formation and oxidative stress of $\beta$-amyloid. J Agric Food Chem 53:8537-8541

43. Kisselev AF, Goldberg AL (2001) Proteasome inhibitors: from research tools to drug candidates. Chem Biol 8:739-758

44. Kwak M-K, Kensler TW (2006) Induction of 26S proteasome subunit PSMB5 by the bifunctional inducer 3-methylcholanthrene through the Nrf2-ARE, but not the AhR/Arnt-XRE, pathway. Biochem Biophys Res Commun 345:1350-1357

45. Kwak M-K, Wakabayashi N, Greenlaw JL, Yamamoto M, Kensler TW (2003) Antioxidants enhance mammalian proteasome expression through the Keap1-Nrf2 signaling pathway. Mol Cell Biol 23:8786-9794

46. Kwak M-K, Huang B, Chang H, Kim J-A, Kensler TW (2007) Tissue specific increase of the catalytic subunits of the $26 \mathrm{~S}$ proteasome by indirect antioxidant dithiolethione in mice: enhanced activity for degradation of abnormal protein. Life Sci 80:2411-2420

47. Kwak M-K, Wakabayashi N, Itoh K, Motohashi H, Yamamoto M, Kensler TW (2003) Modulation of gene expression by cancer chemopreventative dithiolethiones through the Keap1-Nrf2 pathway. J Biol Chem 278:8135-8145

48. Lai M-Y, Hsiu S-L, Tsai S-Y, Hou Y-C, Chao P-DL (2003) Comparison of metabolic pharmacokinetics of baicalin and baicalein in rats. J Pharm Pharmacol 55:205-209

49. Laughton MJ, Evans PJ, Moroney MA, Hoult JRS, Halliwell B (1991) Inhibition of mammalian 5-lipoxygenase and cyclo-oxygenase by flavonoids and phenolic dietary additives. Biochem Pharmacol 42:1673-1681

50. Liu R-M (2002) Down-regulation of $\gamma$-glutamylcysteine synthetase regulatory subunit gene expression in rat brain tissue during aging. J Neurosci Res 68:344-351

51. Liu R-M, Choi J (2000) Age-associated decline in $\gamma$-glutamylcysteine synthetase gene expression in rats. Free Radic Biol Med 28:566-574

52. Lu T, Pan Y, Kao S-Y, Li C, Kohane I, Chan J, Yankner BA (2004) Gene regulation and DNA damage in the ageing human brain. Nature 429:883-891

53. Maher P (2005) The effects of stress and aging on glutathione metabolism. Ageing Res Rev 4:288-314

54. Maher P (2006) A comparison of the neurotrophic activities of the flavonoid fisetin and some of its derivatives. Free Radic Res 40:1105-1111

55. Maher P (2008) Proteasome inhibitors prevent oxidative stressinduced nerve cell death by a novel mechanism. Biochem Pharmacol 75:1994-2006

56. Maher P, Hanneken A (2005) Flavonoids protect retinal ganglion cells from oxidative stress-induced death. Invest Ophthalmol Vis Sci 46:4796-4803

57. Maher P, Akaishi T, Abe K (2006) Flavonoid fisetin promotes ERK-dependent long-term potentiation and enhances memory. Proc Natl Acad Sci USA 103:16568-16573

58. Maher P, Salgado KF, Zivin JA, Lapchak PA (2007) A novel approach to screening for new neuroprotective compounds for the treatment of stroke. Brain Res 1173:117-125

59. Manach C, Williamson G, Morand C, Scalbert A, Remesy C (2005) Bioavailability and bioefficacy of polyphenols in humans. I. Review of 97 bioavailability studies. Am J Clin Nutr 81:230S$242 \mathrm{~S}$

60. Mayr B, Montminy M (2001) Transcriptional regulation by the phosphorylation-dependent factor CREB. Nat Rev Mol Cell Biol 2:599-609 
61. Meister A, Anderson ME (1983) Glutathione. Ann Rev Biochem 52:711-760

62. Middleton E, Kandaswami C, Theoharides TC (2000) The effects of flavonoids on mammalian cells: implications for inflammation, heart disease and cancer. Pharmacol Rev 52:673-751

63. Mosoni L, Breuille D, Buffiere C, Obled C, Mirand PP (2004) Age-related changes in glutathione availability and skeletal carbonyl content in healthy rats. Exp Gerontol 39:203-210

64. Muller FL, Lustgarten MS, Jang Y, Richardson A, Van Remmen $\mathrm{H}$ (2007) Trends in oxidative aging theories. Free Radic Biol Med 43:477-503

65. Myhrstad MCW, Carlsen H, Nordstrom O, Blomhoff R, Moskaug JO (2002) Flavonoids increase the intracellular glutathione level by transactivation of the $\gamma$-glutamylcysteine synthetase catalytical subunit promoter. Free Radic Biol Med 32:386-393

66. Nguyen T, Sherratt PJ, Pickett CB (2003) Regulatory mechanisms controlling gene expression mediated by the antioxidant response element. Annu Rev Pharmacol Toxicol 43:233-260

67. Palomero J, Galan AI, Munoz ME, Tunon MJ, Gonzalez-Gallego J, Jimenez R (2001) Effects of aging on the susceptibility to the toxic effects of cyclosporin $\mathrm{A}$ in rats. Changes in liver glutathione and antioxidant enzymes. Free Radic Biol Med 30:836-845

68. Phillis JW, Horrocks LA, Farooqui AA (2006) Cyclooxygenases, lipoxygenases, and epoxygenases in CNS: their role and involvement in neurological disorders. Brain Res Rev 52:201243

69. Prasain JK, Barnes S (2007) Metabolism and bioavailability of flavonoids in chemoprevention: current analytical strategies and future prospects. Mol Pharm 4:846-864

70. Radi R, Beckman JS, Bush KM, Freeman BA (1991) Peroxynitrite oxidation of sulfhydryls. The cytotoxic potential of superoxide and nitric oxide. J Biol Chem 266:4244-4250

71. Rathbun WB, Murray DL (1991) Age-related cysteine uptake as rate limiting in glutathione synthesis and glutathione half-life in the cultured human lens. Exp Eye Res 53:205-212

72. Rebrin I, Kamzalov S, Sohal RS (2003) Effects of age and caloric restriction on glutathione redox status in mice. Free Radic Biol Med 35:626-635

73. Rivera F, Urbanavicius J, Gervaz E, Morquio A, Dajas F (2004) Some aspects of the in vivo neuroprotective capacity of flavonoids: bioavailability and structure-activity relationship. Neurotox Res 6:543-553

74. Rock RB, Peterson PK (2006) Microglia as a pharmacological target in infectious and inflammatory diseases on the brain. J Neuroimmune Pharmacol 1:117-126

75. Ross JA, Kasum CM (2002) Dietary flavonoids: bioavailability, metabolic effects, and safety. Annu Rev Nutr 22:19-34

76. Sagara Y, Vahnnasy J, Maher P (2004) Induction of PC12 cell differentiation by flavonoids is dependent upon extracellular signal-regulated kinase activation. J Neurochem 90:1144-1155

77. Sandhu SK, Kaur G (2002) Alterations in oxidative stress scavenger system in aging rat brain and lymphocytes. Biogerontology 3:161-173

78. Sato H, Tamba M, Ishii T, Bannai S (1999) Cloning and expression of a plasma membrane cystine/glutamate exchange transporter composed of two distinct proteins. J Biol Chem 274:11455-11458

79. Sawada M, Sawada H, Nagatsu T (2008) Effects of aging on neuroprotective and neurotoxic properties of microglia in neurodegenerative diseases. Neurodegener Dis 5:254-256

80. Shia C-S, Tsai S-Y, Kuo S-C, Hou Y-C, Chao P-DL (2009) Metabolism and pharmacokinetics of 3, 3', 4', 7-tetrahydroxy- flavone (fisetin), 5-hydroxyflavone and 7-hydroxyflavone and antihemolysis effects of fisetin and its serum metabolites. J Agric Food Chem 57:83-89

81. Shukitt-Hale B, Carey AN, Jenkins D, Rabin BM, Joseph J (2007) Beneficial effects of fruit extracts on neuronal function and behavior in a rodent model of accelerated aging. Neurobiol Aging 28:1187-1194

82. Stevenson DE, Cooney JM, Jensen DJ, Wibisono R, Adaim A, Skinner MA, Zhang J (2008) Comparison of enzymically glucuronidated flavonoids with flavonoid aglycones in an in vitro cellular model of oxidative stress protection. In Vitro Cell Dev Biol Anim (Epub)

83. Stolzing A, Widmer R, Jung T, Voss P, Grune T (2006) Tocopherol-mediated modulation of age-related changes in microglial cells: turnover of extracellular oxidized material. Free Radic Biol Med 40:2126-2135

84. Suh JH, Shenvi SV, Dixon BM, Liu H, Jaiswal AK, Liu R-M, Hagen TM (2004) Decline in transcriptional activity of Nrf2 causes age-related loss of glutathione synthesis, which is reversible with lipoic acid. Proc Natl Acad Sci USA 101:33813386

85. Sung B, Pandey MK, Aggarwal BB (2007) Fisetin, an inhibitor of cyclin-dependent kinase 6, down-regulates nuclear factor-kBregulated cell proliferation, antiapoptotic and metastatic gene products through the suppression of TAK-1 and receptor-interacting protein-regulated IkBa kinase activation. Mol Pharmacol 71:1703-1714

86. Tan S, Schubert D, Maher P (2001) Oxytosis: a novel form of programmed cell death. Curr Top Med Chem 1:497-506

87. Torreilles F, Salman-Tabcheh S, Guerin M, Torreilles J (1999) Neurodegenerative disorders: the role of peroxynitrite. Brain Res Brain Res Rev 30:153-163

88. Tully T, Bourtchouladze R, Scott R, Tallman J (2003) Targeting the CREB pathway for memory enhancers. Nat Rev Drug Discov 2:267-277

89. Valerio LG, Kepa JK, Pickwell GV, Quattrochi LC (2001) Induction of human NAD(P)H:quinone oxidoreductase (NQO1) gene expression by the flavonol quercetin. Toxicol Lett 119:4957

90. van Acker SABE, van den Berg D-J, Tromp MNJL, Griffioen DH, van Bennekom WP, van der Vijgh WJF, Bast A (1996) Structural aspects of antioxidant activity of flavonoids. Free Radic Biol Med 20:331-342

91. Vorhees CV, Williams MT (2006) Morris water maze: procedures for assessing spatial and related forms of learning and memory. Nat Protoc 1:848-858

92. Wang L, Tu Y-C, Lian T-W, Hung J-T, Yen J-H, Wu M-J (2006) Distinctive antioxidant and antiinflammatory effects of flavonols. J Agric Food Chem 54:9798-9804

93. Wang Q, Rager JD, Weinstein K, Kardos PS, Dobson GL, Li J, Hidalgo IJ (2005) Evaluation of the MDR-MDCK cell line as a permeability screen for the blood brain barrier. Int $\mathrm{J}$ Pharm 288:349-359

94. Yankner BA, Lu T, Loerch P (2008) The aging brain. Annu Rev Pathol Mech Dis 3:41-66

95. Zeng B-Y, Medhurst AD, Jackson M, Rose S, Jenner P (2005) Proteasomal activity in brain differs between species and brain regions and changes with age. Mech Ageing Dev 126:760-766

96. Zheng LT, Ock J, Kwon B-M, Suk K (2008) Suppressive effects of flavonoid fisetin on lipopolysaccharide-induced microglial activation and neurotoxicity. Int Immunopharmacol 8:484-494 